\title{
Variation in serotonin neurotransmission genes affects neural activation during response inhibition in adolescents and young adults with ADHD and healthy controls
}

\author{
Daan van Rooij, Catharina A. Hartman, Marjolein M.J. van Donkelaar, \\ Janita Bralten, Daniel von Rhein, Marina Hakobjan, Barbara Franke, Dirk J. \\ Heslenfeld, Jaap Oosterlaan, Nanda Rommelse, Jan K. Buitelaar \& Pieter J. \\ Hoekstra
}

To cite this article: Daan van Rooij, Catharina A. Hartman, Marjolein M.J. van Donkelaar, Janita Bralten, Daniel von Rhein, Marina Hakobjan, Barbara Franke, Dirk J. Heslenfeld, Jaap Oosterlaan, Nanda Rommelse, Jan K. Buitelaar \& Pieter J. Hoekstra (2015) Variation in serotonin neurotransmission genes affects neural activation during response inhibition in adolescents and young adults with ADHD and healthy controls, The World Journal of Biological Psychiatry, 16:8, 625-634, DOI: $10.3109 / 15622975.2015 .1067371$

To link to this article: https://doi.org/10.3109/15622975.2015.1067371

+ View supplementary material 둔

Submit your article to this journal $๘$
Published online: 01 Oct 2015.

III Article views: 353

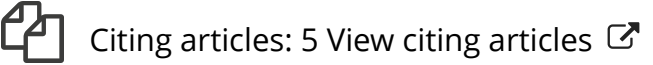




\title{
Variation in serotonin neurotransmission genes affects neural activation during response inhibition in adolescents and young adults with ADHD and healthy controls
}

\author{
DAAN VAN ROOIJ ${ }^{1,2}$, CATHARINA A. HARTMAN ${ }^{1}$, MARJOLEIN M.J. VAN DONKELAAR ${ }^{3}$, \\ JANITA BRALTEN ${ }^{3,4}$, DANIEL VON RHEIN ${ }^{2,4}$, MARINA HAKOBJAN ${ }^{3}$, \\ BARBARA FRANKE ${ }^{3,5}$, DIRK J. HESLENFELD ${ }^{6}$, JAAP OOSTERLAAN ${ }^{6}$, \\ NANDA ROMMELSE ${ }^{7}$, JAN K. BUITELAAR ${ }^{4,7 \#}$ \& PIETER J. HOEKSTRA ${ }^{1 \#}$ \\ ${ }^{1}$ University Medical Center Groningen, Department of Psychiatry, University of Groningen, Groningen, The Netherlands, \\ ${ }^{2}$ Donders Institute for Brain, Cognition and Behaviour, Centre for Cognitive Neuroimaging, Radboud University, \\ Nijmegen, The Netherlands, ${ }^{3}$ Radboud University Medical Center, Donders Institute for Brain, Cognition and Behaviour, \\ Human Genetics Department, Nijmegen, The Netherlands, ${ }^{4}$ Radboud University Medical Center, Donders Institute for \\ Brain, Cognition and Behaviour, Department of Cognitive Neuroscience, Nijmegen, The Netherlands, ${ }^{5}$ Radboud University \\ Medical Center, Donders Institute for Brain, Cognition and Behaviour, Department of Psychiatry, Nijmegen, \\ The Netherlands, ${ }^{6}$ Department of Clinical Neuropsychology, VU University Amsterdam, Amsterdam, The Netherlands, \\ ${ }^{7}$ Karakter Child and Adolescent Psychiatry University Center, Nijmegen, The Netherlands
}

\begin{abstract}
Objectives. Deficits in response inhibition have been associated with attention-deficit/hyperactivity disorder (ADHD). Given the role of serotonin in ADHD and impulsivity, we postulated that genetic variants within the serotonin pathway might influence response inhibition. Methods. We measured neural activation during stop-signal task performance in adolescents with ADHD $(N=185)$, their unaffected siblings $(N=111)$, and healthy controls $(N=124)$, and investigated the relationship of two serotonin gene polymorphisms (the rs6296 SNP of the HTR1B gene and HTTLPR variants of the 5-HTT gene) with the neural correlates of response inhibition. Results. The whole-brain analyses demonstrated large scale neural activation differences in the inferior and medial frontal and temporal/parietal regions of the response inhibition network between the different variants of both the HTR1B and 5HTT genes. Activation in these regions was significantly associated with stop-task performance, but not with ADHD diagnosis or severity. No associations were found between HTR1B and $5 H T T$ variants and ADHD or ADHD-related neural activation. Conclusions. These results provide novel evidence that serotonin may play an important role in the neurobiology of response inhibition. Although response inhibition is strongly linked to ADHD, serotonin linked genetic variants associated with response inhibition and its neural correlates do not explain variance of the ADHD phenotype.
\end{abstract}

Key words: ADHD; response inhibition; $5 H T$; siblings; endophenotype

\section{Introduction}

Serotonin neurotransmission has a link with both cognitive control and impulsivity, one of the defining characteristics of attention-deficit/hyperactivity disorder (ADHD; see for a review Cools et al. 2008). A main cognitive control function is the process of response inhibition, or the ability to withhold, delay, or alter an already initiated response. Response inhibition is associated with impulsivity (Nigg 2000) and has therefore been extensively studied in relation to ADHD (Goos et al. 2009; Crosbie et al. 2013). Recently, neural correlates of response inhibition have been reported as potential endophenotypes for $\mathrm{ADHD}$, going beyond purely behavioural measures (Durston et al. 2006; Van Rooij et al. 2015a).

\#Shared last authors.

Correspondence: Daan van Rooij, Donders Institute, P.O. Box 9104, 6500 HE Nijmegen, The Netherlands. E-mail: d.vanrooij@fcdonders. ru.nl. 
On one hand, evidence for the link between serotonin and impulsivity stems from studies of tryptophan (the 5-HT precursor) depletion. Testing the effects of acute tryptophan depletion in healthy human volunteers demonstrated that tryptophan depletion increased impulsive behaviour (Walderhaug et al. 2002; Finger et al. 2007), but did not alter stop-signal response inhibition performance (Clark et al. 2005). However, tryptophan depletion was shown to be associated with decreased neural activation in the response inhibition network even in the absence of altered behavioural response inhibition performance (Rubia et al. 2005). Thus, these results suggest that neural measures may offer more insight into the mechanisms underlying the influence of serotonin neurotransmission on response inhibition.

Also, genetic studies have indicated that impaired serotonergic transmission is associated with increased impulsivity (Winstanley et al. 2006). A meta-analysis (Gizer et al. 2009) has indicated two serotonin-related gene variants as risk factors for ADHD. The first is the HTTLPR long allele of the 5-HTT (or $S L C 6 A 4)$ serotonin transporter gene. On the other hand, it is the $\mathrm{S}$ allele that has been associated with lower serotonin availability (Heinz et al. 2000). Genetic association studies have also shown inconsistent results for this polymorphism. The $S$ allele of HTTLPR has been linked with heightened impulsivity in healthy participants (Walderhaug et al. 2010). However, other studies have reported no association between the HTTLPR $S$ allele and impulsivity (Baca-Garcia et al. 2004), or even the opposite effect, with increased impulsivity for carriers of the $\mathrm{L}$ allele (Lee et al. 2003).

The second serotoninergic genetic polymorphism implicated in ADHD in the meta-analysis of Gizer et al. (2009) is the rs6296 single nucleotide polymorphism (SNP) G allele in the HTR1B serotonin receptor gene. This $G$ allele is part of a haplotype block causing decreased HTR $1 B$ expression (Duan et al. 2003), leading to decreased serotonin transmission (Sanders et al. 2001; Conner et al. 2010). The rs6296 $\mathrm{G}$ allele has been implicated in both trait impulsivity (Varga et al. 2012) and psychiatric disorders like depression, bipolar disorder, and substance abuse (Huang et al. 2003; Conner et al. 2010; Murphy et al. 2011), suggesting a role for HTR $1 B$ in both cognition and psychiatric disease phenotypes.

Polymorphisms of the serotonin transporter and receptor genes have also been linked to response inhibition performance in healthy participants (i.e., the HT1A C-1019G polymorphism and rs6296, respectively) (Stoltenberg et al. 2006; Beste et al. 2011), and, importantly, have been shown to influence both impulsivity and response inhibition performance in individuals with ADHD (Oades et al. 2008). So far, studies are lacking on the role of $H T R 1 B$ or $5 H T T$ in the neural correlates of response inhibition, both in healthy controls and individuals with ADHD.

Given the previously found associations between serotonin genes, impulsivity, and ADHD, the goal of the current study was to investigate the role of 5HTT and $H T R 1 B$ variants on the neural correlates of response inhibition, behavioural performance, and the clinically defined ADHD phenotype in a sample of adolescents with ADHD, their unaffected siblings, and healthy controls. Inclusion of unaffected siblings enabled us to examine the role of familiality in the distribution of genetic risk factors as well as neural activation patterns. Particularly, we aimed to assess the role of the HTTLPR and rs6296 polymorphisms in this sample using three methods: first, we investigated whole-brain neural activation during response inhibition in relation to these polymorphisms. Second, we investigated if the HTTLPR and rs6296 polymorphisms were associated with ADHD diagnosis and response inhibition performance. Last, we tested if these variants could explain the differences in neural activation in regions that exhibit differential brain responses in ADHD. We expected that the rs6296 and HTTLPR variants associated with higher impulsivity might also influence response inhibition, reflected in decreased activation in the response inhibition network, which in turn might explain variance in the $\mathrm{ADHD}$ phenotype.

\section{Methods}

\section{Participants}

Participants were part of the NeuroIMAGE study, the Dutch follow up of the International Multicenter ADHD Genetics (IMAGE) study. Details concerning informed consent, recruitment, demographics, diagnostics, and testing procedures can be found in the NeuroIMAGE methods publication (Von Rhein et al. 2014). Within the current sample, we included participants with ADHD $(N=184)$, their unaffected siblings $(N=111)$, and healthy controls $(N=124)$. Participant demographics for our study are listed in Table I.

\section{Stop-Signal task}

Response inhibition was measured using a version of the Stop-Signal task (Logan et al. 1984) adapted for functional magnetic resonance imaging (fMRI; Van Meel et al. 2007). Participants were instructed to respond as quickly as possible to a go-signal by choosing the correct response out of two possibilities, unless the go-signal was followed after a short 
Table I. Participant characteristics and task outcomes derived from Stop signal task.

\begin{tabular}{|c|c|c|c|c|c|c|c|c|c|}
\hline & \multicolumn{2}{|c|}{$\begin{array}{l}\text { Participants } \\
\text { with ADHD }\end{array}$} & \multicolumn{2}{|c|}{$\begin{array}{l}\text { Unaffected } \\
\text { Siblings }\end{array}$} & \multicolumn{2}{|c|}{ Controls } & Wald-chi ${ }^{2}$ & $P$ value & Between group effects \\
\hline Males & \multicolumn{2}{|c|}{$69.7 \%$} & \multicolumn{2}{|c|}{$56.7 \%$} & \multicolumn{2}{|c|}{$55.6 \%$} & 28.1 & $<0.001$ & $\mathrm{ADHD}>($ Sibs $=$ Controls $)$ \\
\hline Stimulant Medication use & \multicolumn{2}{|c|}{$53.8 \%$} & \multicolumn{2}{|c|}{$2.9 \%$} & \multicolumn{2}{|c|}{$0 \%$} & 189.54 & $<0.001$ & $\mathrm{ADHD}>($ Sibs $=$ Controls $)$ \\
\hline Comorbid ODD ${ }^{\mathrm{a}}$ & \multicolumn{2}{|c|}{$29.9 \%$} & \multicolumn{2}{|c|}{$3.6 \%$} & \multicolumn{2}{|c|}{$0 \%$} & 67.686 & $<0.001$ & $\mathrm{ADHD}>($ Sibs $=$ Controls $)$ \\
\hline \multirow{2}{*}{ Comorbid CD } & \multicolumn{2}{|c|}{$6.5 \%$} & \multicolumn{2}{|c|}{$0 \%$} & \multicolumn{2}{|c|}{$0 \%$} & 15.626 & $<0.001$ & $\mathrm{ADHD}>($ Sibs $=$ Controls $)$ \\
\hline & Mean & SD & Mean & SD & Mean & $\mathrm{SD}$ & & & \\
\hline $\mathrm{ADHD}^{\mathrm{b}}$ symptoms & 12.9 & 3.1 & 1.3 & 3.4 & 0.6 & 1.5 & 2427 & $<0.001$ & $\mathrm{ADHD}>($ Sibs $=$ Controls $)$ \\
\hline Age (years) & 17.3 & 3.2 & 17.3 & 4 & 16.5 & 3.3 & 1.6 & 0.44 & - \\
\hline Estimated $\mathrm{IQ}^{\mathrm{c}}$ & 95.3 & 16.8 & 102.4 & 15.9 & 107.1 & 14.5 & 38.2 & $<0.001$ & ADHD $<$ Sibs $<$ Controls) \\
\hline Education (years) & 12.82 & 2.14 & 12.82 & 2.22 & 13.52 & 1.91 & 6.387 & 0.041 & $(\mathrm{ADHD}=\mathrm{Sibs})<$ Controls \\
\hline $\operatorname{SSRT}(\mathrm{ms})^{\mathrm{d}}$ & 268.1 & 59.4 & 254.1 & 49.0 & 258.2 & 52.6 & 6.421 & 0.04 & $\mathrm{ADHD}>($ Sibs $=$ Controls $)$ \\
\hline $\mathrm{ICV}^{\mathrm{d}}$ & 112 & 38.3 & 93.2 & 36.7 & 82.2 & 30.8 & 37.801 & $<0.001$ & ADHD $>$ Sibs $>$ Controls \\
\hline Errors $(n)^{\mathrm{d}}$ & 6.3 & 7.6 & 4.2 & 5.6 & 3.1 & 3.5 & 16.884 & $<0.001$ & ADHD $>$ Sibs $>$ Controls \\
\hline
\end{tabular}

Note: ADHD, attention deficit/hyperactivity disorder; ODD, oppositional defiant disorder; CD, conduct disorder; SSRT, stop-signal reaction time; ICV, intra-individual coefficient of variance; Errors, number of errors on go-trials. Bold values indicate significant effects. ${ }^{a} \mathrm{ODD}$ and CD diagnosis was based on K-SADS structured psychiatric interviews. ${ }^{\mathrm{b}} \mathrm{ADHD}$ diagnosis was based on K-SADS structured psychiatric interviews and Conners' questionnaires. 'Estimated IQ was based on two subtests of the Wechsler Intelligence Scale for Children (WISC) or Wechsler Adult Intelligence Scale (WAIS-III). ${ }^{\mathrm{d}}$ Task effects for the stop-task derived from Generalized Estimate Equation model, corrected for familiality, gender, age, and IQ.

interval by a stop-signal ( $25 \%$ of trials), in which case they were instructed to withhold their response. By varying the delay between go- and stop-signal, it was possible to derive the main outcome measure of the task, the Stop-Signal Reaction Time (SSRT), which reflects the time necessary for a participant to successfully inhibit their response in $50 \%$ of the stop-trials. Secondary outcome measures were the number of omission and commission (i.e., a wrong button response) errors on go-trials (errors) and the Intra-individual component of variation (ICV), derived by dividing the reaction time variability by the mean reaction time over all go-trials. The task consisted of a total of four blocks of 60 trials, separated by 1 -min intervals.

Task outcome analyses were performed in SPSS (version 19.0, SPSS Inc.), General Estimated Equations (GEE) regression models were used to correct for familial relations between siblings. Separate regression models were executed for SSRT, ICV Errors, and MRT, with age, gender, and IQ added as covariates. A significance threshold of 0.05 was entrained for all analyses.

\section{Genotyping}

An extensive description of DNA extraction and genotyping of the HTTLPR VNTR in IMAGE is provided elsewhere (Brookes et al. 2006). The rs6296 SNP was genotyped using KASPar analysis at the Radboud University Medical Center, details can be found in the Supplementary Information (SI) available online at http://dx.doi.org/10.3109/15622975. 2015.1067371 .

\section{fMRI acquisition and analysis}

FMRI data were collected at two sites using similar Siemens Scanners and identical coils and protocols, and were processed using FSL FEAT (FMRIB's Software Library, www.fmrib.ox.ac.uk/fsl; version 6.0). Details regarding acquisition, preprocessing and first-level analysis can be found in the SI available online at http://dx.doi.org/10.3109/15622975.2 015.1067371 .

\section{Genetic effects on ADHD diagnosis and task performance}

The diagnostic group factor consisted of three groups of interest, i.e., participants with ADHD, unaffected siblings, and unrelated controls. The effects of diagnosis and behavioural response inhibition were investigated using chi-squared statistics and analysis of variance respectively (see Tables II and III).

\section{Role of genetic variants on whole-brain activation in the combined ADHD-control sample}

To investigate the effect of each genetic variant on task activation at the whole brain level, two separate higher-level analyses were conducted. An F-contrast was constructed for each polymorphism, treating the three possible rs6296 genotypes or the three HTTLPR genotypes as primary between-participant factor. ADHD diagnosis was entered as a second factor in order to investigate possible mediation or interaction effects. Age, IQ, gender, and scan site were added as nuisance regressors in all group-level analyses. Statistical inference was done after correction for multiple comparisons, thresholding at a 
Table II. Distribution of genotypes per diagnostic group.

\begin{tabular}{|c|c|c|c|c|c|c|c|c|c|c|c|c|}
\hline \multirow[b]{2}{*}{ Gene } & \multirow[b]{2}{*}{ Polymorphism } & \multirow{2}{*}{$\begin{array}{c}\text { Risk } \\
\text { genotype }\end{array}$} & \multirow[b]{2}{*}{ MAF } & \multirow{2}{*}{$\frac{\text { HWE }}{P \text { value }}$} & \multicolumn{2}{|c|}{ ADHD } & \multicolumn{2}{|c|}{ Siblings } & \multicolumn{2}{|c|}{ Control } & \multirow[b]{2}{*}{ Odds ratio ${ }^{a}$} & \multirow[b]{2}{*}{$P$ value } \\
\hline & & & & & Risk & No risk & Risk & No risk & Risk & No risk & & \\
\hline 5-HTT & HTTLPR & LL & 0.37 & 0.78 & 65 & 94 & 43 & 58 & 30 & 71 & $1.637(0.962-2.78)$ & 0.217 \\
\hline HTR1B & rs6296 & $\mathrm{CC}$ & 0.26 & 0.29 & 67 & 90 & 44 & 54 & 42 & 52 & $1.085(0.648-1.816)$ & 0.517 \\
\hline
\end{tabular}

Note: MAF, minor allele frequency; HWE, Hardy-Weinberg equilibrium. ${ }^{a}$ Odds Ratio illustrate the relative distribution of genotypes between participants with ADHD and healthy controls.

voxel-level $(Z<2.3)$ using Gaussian random field (GRF) theory-based cluster statistics at $P<0.05$ (FSL cluster; Woo et al. 2014). Post-hoc tests were performed for beta values from clusters showing significant main effects of genetic variants to specify the direction of the genetic effects and to investigate potential effects of diagnostic group. Post-hoc tests were performed using GEE analyses in SPSS, correcting for familial dependency between siblings. Additionally, correction for multiple comparisons between nodes was done using Bonferroni-Holm (Holm 1979) correction.

Additional models were run to associate the extracted beta-values with stop-task performance as well as with the number of ADHD symptoms. Besides the above-mentioned covariates, family membership was added as a between-participant factor in all above-mentioned models to account for the family structure of our data.

\section{Sensitivity analyses}

Sensitivity analyses were performed using similar GEE models to investigate any potential confounding effects of age, gender, IQ, and scanner-site on whole-brain activation, together with tests investigating the potential effects of stimulant medication use and duration (as measured by self-report questionnaire and pharmacist prescription data), as well as the potential effects of comorbid oppositional defiant disorder and conduct disorder.

\section{Genetic effects on diagnosis-sensitive task responses}

To investigate genetic effects on regions that exhibit differential brain responses in ADHD, we applied region of interest (ROI) analyses. For the three main task contrasts, namely failed stop-go, successful stop-go and failed-successful stop trials, ROIs were defined functionally by calculating an Fcontrast for the diagnostic group $\times$ task effects on neural activation across all participants (see Supplementary I available online at http://dx.doi.org/10. 3109/15622975.2015.1067371, or Van Rooij et al. 2015a). Beta values from these ROIs were exported from the individual contrast maps and subsequently used to test the effect of the three possible HTTLPR or rs6296 variants. We used GEE models for each ROI separately with the same predictors as mentioned above. Likewise, familial relatedness was entered as a random factor to correct for nonindependence of the data. Gender, age, IQ, and scansite were added as covariates. $P$-values were corrected for multiple comparisons using Bonferroni-Holm correction (Holm 1979).

\section{Results}

Genetic effects on diagnostic status and task outcome measures

The distribution of the risk variants did not differ significantly between participants with ADHD, their unaffected siblings, and healthy controls (see Table II). No significant relations between any of the risk variants and task outcome measures were observed, nor were there any main effects of (or interactions with) age, gender, or IQ (see Table III).

\section{Genetic effects on whole-brain fMRI activation}

Both HTTLPR and rs6296 genotype significantly influenced the neural activation in the successful

Table III. Relationships between gene variants and stop-task outcome measures.

\begin{tabular}{|c|c|c|c|c|c|c|c|c|}
\hline \multirow[b]{2}{*}{ Gene } & \multirow[b]{2}{*}{ Polymorphism } & \multirow[b]{2}{*}{ Risk genotype } & \multicolumn{2}{|c|}{ SSRT $^{\mathrm{a}}$} & \multicolumn{2}{|c|}{$\mathrm{ICV}^{\mathrm{a}}$} & \multicolumn{2}{|c|}{ Errors $^{\mathrm{a}}$} \\
\hline & & & $\mathrm{Chi}^{2}$ & $P$ value & $\mathrm{Chi}^{2}$ & $P$ value & $\mathrm{Chi}^{2}$ & $P$ value \\
\hline 5-HTT & HTTLPR & $\mathrm{LL}$ & 0.751 & 0.687 & 0.685 & 0.71 & 3.619 & 0.057 \\
\hline$H T R 1 B$ & rs6296 & $\mathrm{CC}$ & 1.016 & 0.602 & 1.696 & 0.428 & 0.779 & 0.677 \\
\hline
\end{tabular}

Note: SSRT, stop-signal reaction time; ICV, intra-individual coefficient of variance; Errors, number of omission and commission errors on go-trials. Bold values indicate significant outcomes. ${ }^{\mathrm{a}} \mathrm{Gene}$ effects on the stop-task outcome measures were derived from generalized estimating equation model corrected for familiality, age, gender and IQ. 
stop-go and failed stop-go contrasts. We found differential activation for the HTTLPR genotypes in the left frontal pole, right cerebellum, and right inferior/orbitofrontal gyrus during successful stop trials. During failed stop trials, nodes of differential activation were found in the right inferior frontal gyrus, frontal pole, cingulate gyrus, and the brainstem (see Figure 1). Post-hoc tests indicated that in every case the effects were driven by altered neural activation in the SS genotype as compared to the SL and LL genotype; with the SS genotype showing decreased activation in the frontal nodes and increased activation in posterior nodes as compared to the other two genotypes (see Table IV).

Rs6296 genotype was associated with differential activation in anterior cingulate, occipital, inferior temporal, and cerebellar regions during successful stop trials. During failed stops, inferior and superior frontal gyrus, superior parietal gyrus, occipital cortex, and precuneus were differentially active (see Figure 2). Post-hoc tests indicated that these group effects were mainly driven by the difference between the CC genotype and CG and/or GG genotype. However, the direction of these effects was inconsistent, with both increased and decreased activation for the CC genotype being observed in frontal and posterior nodes (see Table IV).
Role of genetic effects in whole-brain fMRI activation, stop-task performance, and ADHD severity

During successful response inhibition, the right inferior/orbitofrontal area that was differentially activated for the different HTTLPR genotypes was also associated with SSRTs $\left(\beta=-0.113, \chi^{2}=9.511\right.$, $P=0.002$ ), indicating better response inhibition with increased activation in this node. Both the right inferior/orbitofrontal area and left frontal pole were additionally associated with error rates $(\beta=0.921$, $\chi^{2}=6.986, P=0.008 ; \beta=0.95, \chi^{2}=9.217, P=0.002$, respectively), both indicating increased error rates with higher neural activation in these clusters (see Supplementary I available online at http://dx.doi. org/10.3109/15622975.2015.1067371).

Neural activation in the right anterior cingulate gyrus that showed differential activation for the HTR $1 B$ genotypes was negatively correlated with SSRT $\left(\beta=-0.061, \chi^{2}=9.083, P=0.003\right)$ during successful inhibitions, indicating increased inhibition performance with higher anterior cingulate activation. No other significant correlations between neural activation and task performance survived correction for multiple comparisons (see Supplementary I available online at http://dx.doi. org/10.3109/15622975.2015.1067371).

Though no direct effect of HTTLPR and HTR1B genotypes on SST performance were detected, additional mediation analyses (Hayes 2013) were
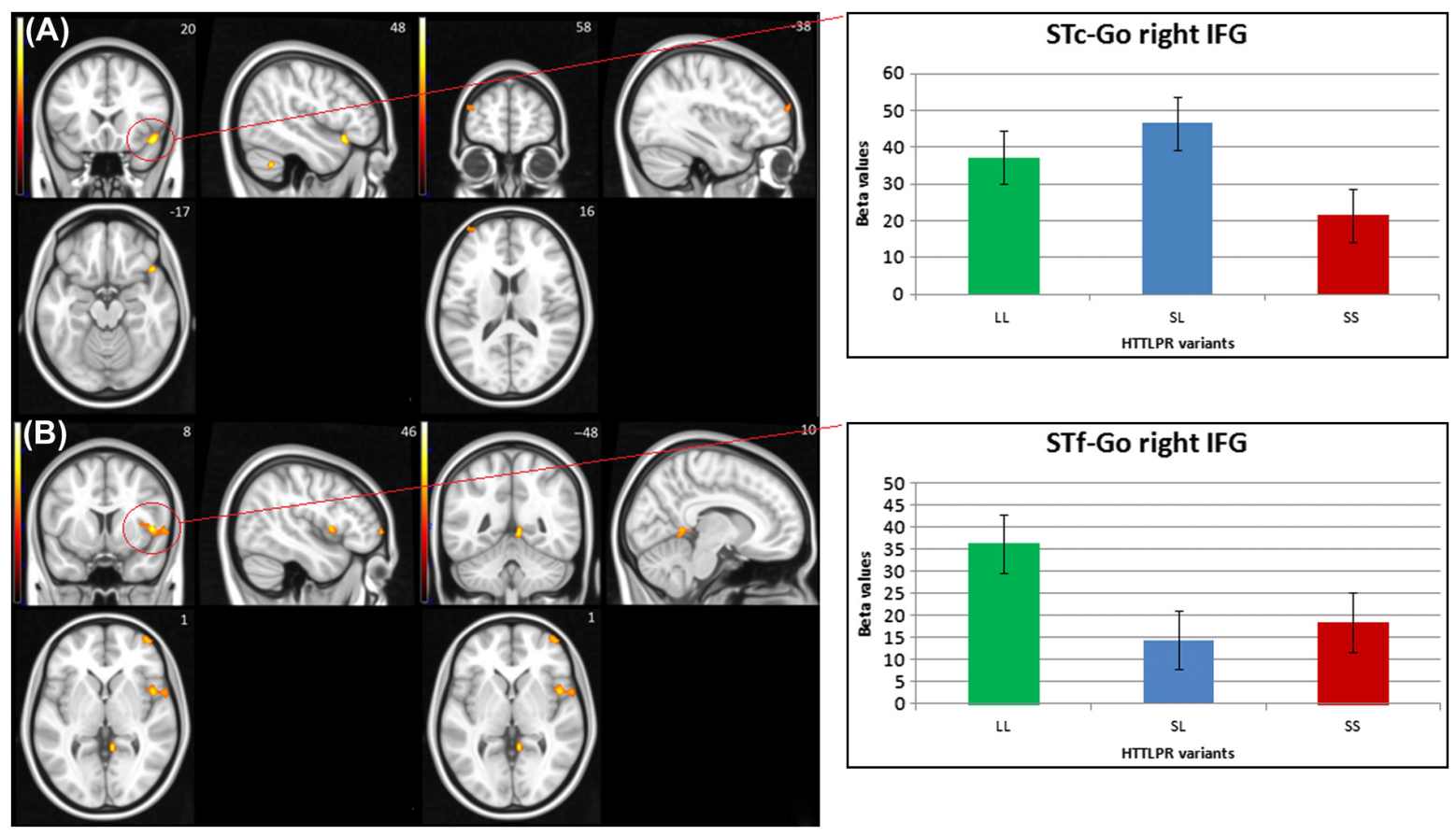

Figure 1. Effects of the HTTLPR variant on neural activation during successful-stop contrast (A) and failed-stop contrast (B). Right side of the image depicts right side of the brain. 
Table IV. Role of HTTLPR genotypes in brain activation during the Stop Signal Task.

\begin{tabular}{|c|c|c|c|c|c|c|c|c|}
\hline & Side & \#Voxels & $P^{b}$ & $F$ & $x$ & $y$ & $z$ & Group effect \\
\hline \multicolumn{9}{|c|}{ Effects of HTTLPR on neural activation } \\
\hline \multicolumn{9}{|c|}{ Successful-stop contrast ${ }^{a}$} \\
\hline Frontal pole & $\mathrm{L}$ & 113 & $<0.001$ & 2.89 & -36 & 62 & 16 & $\mathrm{SS}<\mathrm{SL}=\mathrm{LL}$ \\
\hline Cerebellum & $\mathrm{R}$ & 89 & $<0.01$ & 3.71 & 48 & -54 & -40 & $\mathrm{SS}>\mathrm{SL}=\mathrm{LL}$ \\
\hline Inferior/Orbitofrontal gyrus & $\mathrm{R}$ & 90 & $<0.01$ & 3.95 & 46 & 20 & -14 & $\mathrm{SS}<\mathrm{SL}=\overline{\mathrm{LL}}$ \\
\hline \multicolumn{9}{|l|}{ Failed-stop contrast } \\
\hline Inferior frontal gyrus & $\mathrm{R}$ & 195 & $<0.0001$ & 3.81 & 48 & 8 & 4 & $\mathrm{SS}<\mathrm{SL}=\mathrm{LL}$ \\
\hline Frontal pole & $\mathrm{R}$ & 140 & $<0.001$ & 3.29 & 42 & 58 & 0 & $\mathrm{SS}<\mathrm{SL}=\mathrm{LL}$ \\
\hline Cingulate gyrus & $\mathrm{R}$ & 113 & $<0.01$ & 3.69 & 6 & -46 & -2 & $\mathrm{SS}>\mathrm{SL}=\mathrm{LL}$ \\
\hline Brainstem & $\mathrm{L} / \mathrm{R}$ & 113 & $<0.01$ & 4.09 & -2 & -32 & -20 & $\mathrm{SS}>\mathrm{SL}=\mathrm{LL}$ \\
\hline \multicolumn{9}{|c|}{ Effects of rs6296 on neural activation } \\
\hline \multicolumn{9}{|c|}{ Successful-stop contrast ${ }^{a}$} \\
\hline Cerebellum & $\mathrm{L}$ & 246 & $<0.0001$ & 3.52 & -32 & -48 & -28 & $\mathrm{CC}>\mathrm{GC}=\mathrm{GG}$ \\
\hline Lateral occipital cortex & $\mathrm{L}$ & 190 & $<0.0001$ & 3.4 & -42 & -90 & -4 & $\mathrm{CC}>\mathrm{GC}$ \\
\hline Anterior cingulate gyrus & $\mathrm{R}$ & 183 & $<0.0001$ & 3.73 & 16 & 42 & 8 & $\mathrm{CC}<\mathrm{GC}=\mathrm{GG}$ \\
\hline Cerebellum & $\mathrm{R}$ & 146 & $<0.0001$ & 3.61 & 26 & -38 & -50 & $\mathrm{CC}>\mathrm{GC}=\mathrm{GG}$ \\
\hline Lateral occipital cortex & $\mathrm{L}$ & 121 & $<0.001$ & 3.31 & -42 & -72 & -16 & $\mathrm{CC}<\mathrm{GC}$ \\
\hline Inferior temporal gyrus & $\mathrm{L}$ & 90 & $<0.05$ & 3.09 & -48 & -70 & 26 & $\mathrm{CC}<\mathrm{GC}$ \\
\hline \multicolumn{9}{|l|}{ Failed-stop contrast } \\
\hline Precuneus & $\mathrm{L} / \mathrm{R}$ & 348 & $<0.0001$ & 3.7 & -2 & -62 & 16 & $\mathrm{CC}<\mathrm{GC}<\mathrm{GG}$ \\
\hline Lateral occipital cortex & $\mathrm{R}$ & 223 & $<0.0001$ & 3.45 & 54 & -68 & 32 & $\mathrm{CC}<\mathrm{GC}=\mathrm{GG}$ \\
\hline Superior frontal gyrus & $\mathrm{L}$ & 162 & $<0.0001$ & 3.39 & -6 & 54 & 28 & $\mathrm{CC}<\mathrm{GC}=\mathrm{GG}$ \\
\hline Superior parietal lobe & $\mathrm{L}$ & 131 & $<0.001$ & 3.93 & -24 & -54 & 48 & $\mathrm{CC}=\mathrm{GG}>\mathrm{GC}$ \\
\hline Inferior frontal gyrus & $\mathrm{R}$ & 116 & $<0.01$ & 2.98 & 60 & 20 & 32 & $\mathrm{CC}>\mathrm{GC}=\mathrm{GG}$ \\
\hline
\end{tabular}

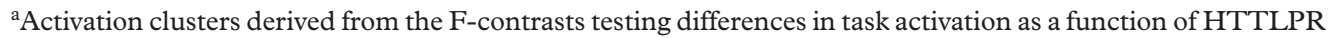
genotype (SS vs. SL vs. LL) or rs6296 genotype (CC vs. CG vs. GG) over all participants, including gender, IQ, age and scan-site as covariates. ${ }^{\mathrm{b}}$ Correction for multiple comparisons in FSL FEAT was done using a cluster threshold of $Z>2.3$ and a significance threshold of $P<0.05$ corrected. ${ }^{\circ}$ Group effects are derived from post-hoc analyses, corrected for familiality.
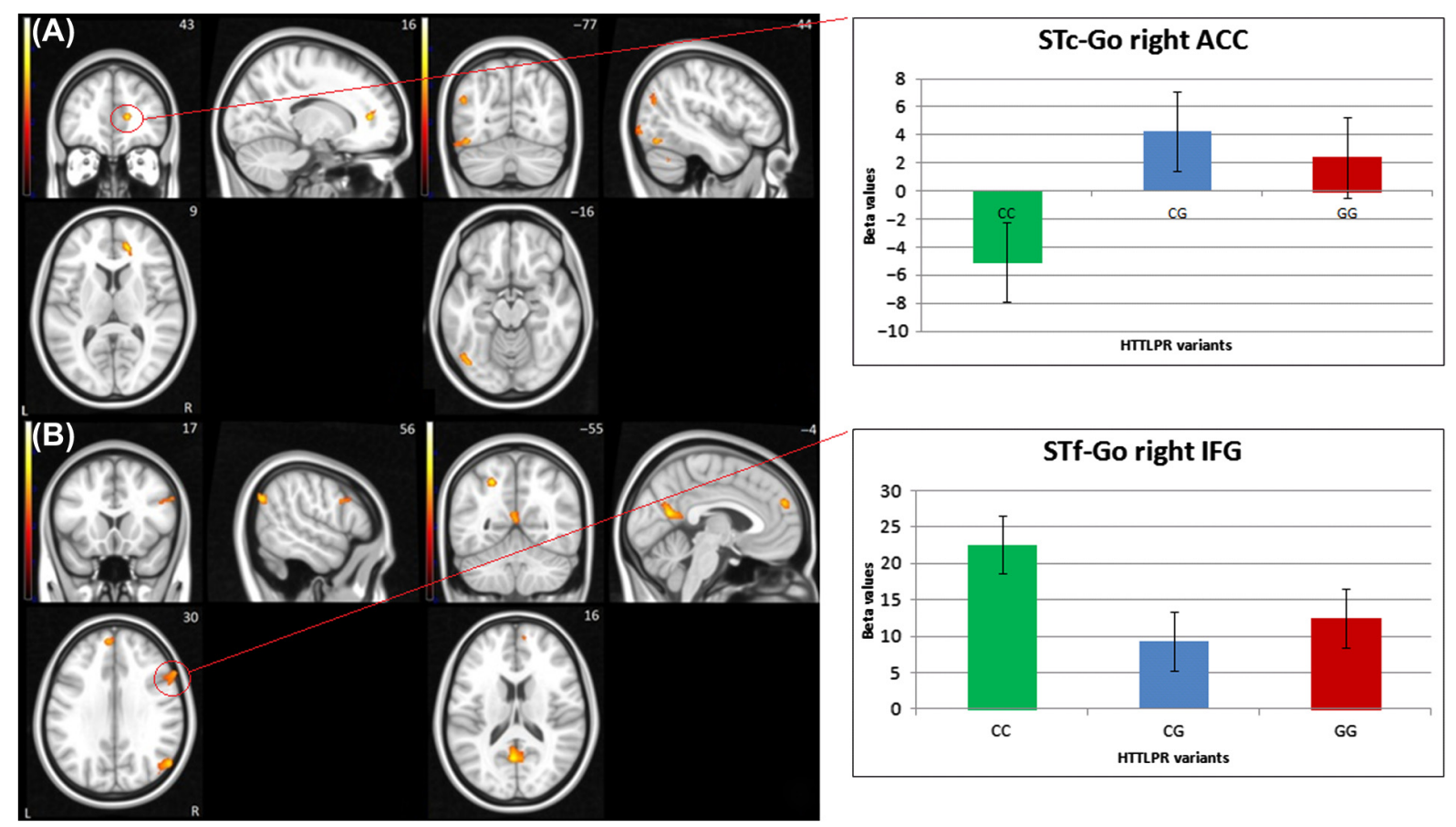

Figure 2. Effects of the rs6296 variant on neural activation from the successful-stop contrast (A) and failed-stop contrast (B). Right side of the image depicts right side of the brain. 
performed to further explore whether the association between the neural activation and SST performance described above was mediated by the genetic variants. No evidence of mediation effects was detected. No significant associations were found between the activation in neural nodes indicated in the whole-brain analysis and either ADHD diagnosis (ADHD, unaffected sibling, or control), nor the number of ADHD symptoms, indicating no relation between $\mathrm{ADHD}$ status and the effects of rs6296 and HTTLPR on neural activation.

\section{Influence of covariates on whole-brain fMRI activation}

To investigate whether the whole-brain activation was influenced by age, gender, IQ, scan site, medication use, and comorbid disorders, several post-hoc analyses were performed. No main or interaction effects of IQ, gender, or scan-site were detected, indicating that these variables did not influence the reported genetic effects on fMRI activation. The activation in the right inferior frontal and frontal pole areas where differential effects of the HTTPLR genotypes were observed during failed inhibitions also showed a main effect of age $(\beta=-1.197, P=0.011 ; \beta=2.637, P=0.005$, respectively), indicating a general decrease of activation in these nodes with increased age. However, there was no interaction with the gene effect in the same location, indicating that both effects occur independently. No other effects of age were observed.

The effects of medication were assessed by incorporating both medication use and duration of use in post-hoc analyses, as were the effects of comorbid diagnoses of oppositional defiant disorder and conduct disorder. None of the medication and comorbidity factors showed main effects or interaction with Genetic effects on differential fMRI activation between diagnostic groups

The main diagnostic group contrast on neural activation during the stop-task indicated differential activation between participants with ADHD, unaffected siblings, and controls in a range of nodes in inferior and superior frontal, anterior cingulate, and temporal/parietal areas. Details regarding these ROIs can be found in (Van Rooij et al. 2015a) as well as in the SI. None of the multivariate tests indicated main (neither with nor without incorporation of the diagnostic effect) or interaction effects with group of rs6296 or HTTLPR genotypes on the neural activation in these ROIs (see Supplementary I available online at http://dx.doi.org/10.3109/15622975. 2015.1067371).

\section{Discussion}

In the present study, we investigated the effects of two genetic variants, HTTLPR in the serotonin transporter gene and rs6296 in the serotonin receptor gene $H T R 1 B$ on response inhibition performance and its underlying neural activation patterns in a cohort consisting of participants with and without $\mathrm{ADHD}$. We provide for the first time direct evidence for a genetically driven effect of serotonin transmission on the neural correlates of response inhibition.

The first part of this study was to test the effects of two genetic variants within $5 H T T$ and $H T R 1 B$ on whole-brain activation during response inhibition. These analyses indicated effects of HTTLPR in the frontal nodes of the response inhibition network, as well as in more posterior nodes like the cerebellum and cingulate cortex. Specifically, decreased neural activation was observed in individuals with the SS genotype in the right inferior frontal gyrus and frontal poles; the former is recognized as an essential node of the response inhibition network (Aron and Poldrack 2006; Chambers et al. 2009). On the other hand, increased neural activation in individuals with the SS genotype was observed in the cerebellum, cingulate cortex, and brainstem. Lower activation in the right inferior frontal region was associated with decreased response inhibition performance during successful stop-trials, although lower activation in the same region as well as in the frontal pole were also associated with lower error rates on go-trials, suggesting a possible deficit in response inhibition, but an increase in general attention performance on go-trials in individuals with the SS genotype (Esterman et al. 2014).

Our results indicate that the effect of HTTLPR on neural activation is driven by the SS genotype, which showed less activity in the frontal response inhibition nodes and more in the posterior areas. The relations between neural activation and stop-task performance further indicate that these frontal areas are directly involved in response inhibition and attentional performance; although no direct effect of HTTLPR genotype on performance was observed. Taken together, these findings might indicate a posterior shift of neural activation in individuals with the SS genotype, possibly compensating for decreased activation of the main response inhibition network. Previous studies show decreased serotonin transporter expression in individuals with the SS genotype, signaling higher serotonin availability (Lesch et al. 1996; Heinz et al. 2000). These findings may indicate a relation between serotonin availability, decreased response inhibition, and increased impulsivity (Walderhaug et al. 2010). However, this would be in conflict with the meta- 
analytic findings marking the $\mathrm{L}$ carriers of the HTTLPR as an ADHD risk-group, given that decreased neural activation in frontal nodes during response inhibition has usually been associated with ADHD severity, including in the current sample (Van Rooij et al. 2015a), although a study also reported an association between the $S$ allele of the HTTLPR and adults with ADHD (Landaas et al. 2010). Another study compared different types of impulsivity paradigms in rats, demonstrating that tryptophan depletion may influence reactivity on gotrials, but not stop-signal reaction times in a go/ no-go task. This indicates that while the delay discounting aspect of impulsivity may have been affected, response inhibition was not (Eagle et al. 2009). These findings may explain our current effects of HTTLPR on neural activation, which showed opposite effects on neural activation levels, error rates, and SSRTs. This dissociation between different aspects of impulsivity will need to be further investigated to fully understand the relation between serotonin, impulsivity, and ADHD.

The effect of rs6296 showed a similar distribution across frontal-parietal, occipital, and cerebellar nodes, equally indicating relatively widespread differential activation. The GG genotype, considered an ADHD risk factor (Gizer et al. 2009), showed increased activation in occipital, temporal/parietal, superior frontal, and cingulate regions, with decreased activation in cerebellar and inferior frontal areas. Of those, activation in anterior-cingulate regions was significantly associated with SSRT length, indicating higher activation levels correlated with better inhibition performance. Temporal parietal, superior frontal, and cingulate regions have all been implicated in attentional control and action monitoring processes necessary for response inhibition (Bekker et al. 2005; Fassbender et al. 2006; van Meel et al. 2007). In addition, previous studies have suggested the involvement of a separate frontal-thalamo-cerebellar pathway involved in inhibitory control (Rubia et al. 2007). The current results may suggest decreased activation in the frontal-cerebellar pathway in G allele carriers, compensated by increased activation in attentional of topdown control areas. We postulate that the utilization of compensatory or alternative strategies using attention resources may explain the lack of direct effects of rs6296 on stop-task performance.

In the second part of the study, we investigated whether variants in the $5 H T T$ and HTR $1 B$ genes were associated with $\mathrm{ADHD}$ diagnosis, response inhibition, or whether previous outcomes detailing the influence of ADHD on neural activation during response inhibition were related to variants in the 5HTT and HTR $1 B$ genes. We found that the HTTLPR and rs6296 variants were not associated with ADHD diag- nosis, nor did they influence the ADHD effect on behavioural or neural measures of response inhibition. These findings therefore suggest there is no direct causal pathway between the genetic variants investigated, response inhibition, and the ADHD phenotype. Results fit within the mediational endophenotype model as discussed in the conceptual review by Kendler and Neale (2010). In this model a relation exists between the genes and endophenotype, as well as between the endophenotype and disease, without a necessary relation between genes and disease. This is specifically true in case of relatively limited effect sizes, in which case the direct association between gene variance and phenotype may be overshadowed by noise, while associations with the neural endophenotype can still be observed. While in our previous publications we found support for the endophenotype to disease relation (Van Rooij et al. 2015a, 2015b), the current results support the relation between genes and the endophenotype. This illustrates how the incorporation of a neural endophenotype allows us to study potential relations between genes and disease phenotype that would otherwise be invisible.

\section{Limitations}

There may be limitations to the current study that should be taken into consideration when interpreting the results. First, we may not have missed a possible relation between serotonin-related gene variants and the ADHD phenotype since we tested only a small part of the functional variants related to the serotonin system. Recent studies have suggested that the cumulative variance across a large number of genes within a single pathway may offer additional explanatory power over the single gene variant approaches (Bralten et al. 2013). Future research should consider a broader scope of functional gene variants across neurotransmitter systems that may be required to fully establish or dissociate the genetic links between response inhibition and ADHD. Second, response inhibition and the variants HTTPLR and rs6296 from the 5-HTT and HTR1B genes have been implicated in a wide range of psychiatric disorders including depression, bipolar disorder, anxiety, and substance abuse disorder (Lesch et al. 1996; Huang et al. 2003; Cho et al. 2005). This may indicate possible shared genetic and neural underpinnings of different psychiatric disorders. The abovementioned findings suggest that diagnostic boundaries between psychiatric disorders may not necessarily represent underlying genetic mechanisms (Lee et al. 2013); and the current findings suggest that the use of neurobiological constructs may provide more specific targets for genetic studies than diagnostic phenotypes.

Future studies should take these limitations into consideration, and aim to broaden the scope of both 
genetic variants and phenotypes incorporated in these studies.

\section{Conclusions}

To summarize, whole-brain analysis of neural activation indicated a broad pattern of differential neural activation in frontal-parietal, cerebellar, and occipital areas during response inhibition associated with HTTLPR and rs6296. Activation in these nodes was related to response inhibition performance, but independent of ADHD diagnosis and severity. These results demonstrate the effect of the HTTLPR and rs6296 variants on the behavioural and neural correlates of response inhibition. Since there were no direct associations between the genetic variants and task performance, neural correlates may be a more sensitive measure of genotype effects than solely behavioural or clinically defined phenotypes.

\section{Acknowledgements}

We acknowledge the department of Pediatrics of the VU University Medical Center for having the opportunity to use the mock scanner for preparation of our participants. We thank Roshan Cools for her invaluable advice and input in this manuscript.

\section{Statement of Interest}

This work was supported by NIH Grant R01MH62873 (to Stephen V. Faraone), NWO Large Investment Grant 1750102007010 and NWO Brain \& Cognition an Integrative Approach grant (433-09-242) (to Jan Buitelaar), and grants from Radboud University Nijmegen Medical Center, University Medical Center Groningen and Accare, and VU University Amsterdam.

Jan K. Buitelaar has been in the past 3 years a consultant to/member of advisory board of/and/or speaker for Janssen Cilag BV, Eli Lilly, Bristol-Myer Squibb, Shering Plough, UCB, Shire, Novartis, and Servier. He is not an employee or stock shareholder of any of these companies. In the past 3 years, Jaap Oosterlaan had an investigator-initiated grant from Shire pharmaceuticals and Pieter J. Hoekstra an investigator-initiated grant from Shire and was a member of the advisory board of Eli Lilly and Shire.

\section{References}

Aron A, Poldrack R. 2006. Cortical and subcortical contributions to stop signal response inhibition: role of the subthalamic nucleus. J Neurosci 26:2424-2433.

Baca-Garcia E,Vaquero C, Diaz-Sastre C, García-Resa E, Saiz-Ruiz J, Fernández-Piqueras J, et al. 2004. Lack of association between the serotonin transporter promoter gene polymorphism and impulsivity or aggressive behavior among suicide attempters and healthy volunteers. Psychiatry Res 126:99-106.
Bekker EM, Kenemans JL, Hoeksma MR, Talsma D, Verbaten MN. 2005. The pure electrophysiology of stopping. Int J Psychophysiol 55:191-198.

Beste C, Domschke K, Radenz B, Falkenstein M, Konrad C. 2011. The functional 5-HT1A receptor polymorphism affects response inhibition processes in a context-dependent manner. Neuropsychologia 49:2664-2672.

Bralten J, Franke B, Waldman I. 2013. Candidate genetic pathways for Attention-Deficit/Hyperactivity Disorder (ADHD) show association to hyperactive/impulsive symptoms in children with ADHD. J Am Acad Child Adolesc Psychiatry 52:1204-1212.

Brookes K-J, Mill J, Guindalini C, Curran S, Xu X, Knight J, et al. 2006. A common haplotype of the dopamine transporter gene associated with attention-deficit/hyperactivity disorder and interacting with maternal use of alcohol during pregnancy. Arch Gen Psychiatry 63:74-81.

Chambers CD, Garavan H, Bellgrove MA. 2009. Insights into the neural basis of response inhibition from cognitive and clinical neuroscience. Neurosci Biobehav Rev 33:631-646.

Cho HJ, Meira-Lima I, Cordeiro Q, Michelon L, Sham P, Vallada H, et al. 2005. Population-based and family-based studies on the serotonin transporter gene polymorphisms and bipolar disorder: a systematic review and meta-analysis. Mol Psychiatry 10:771-781.

Clark L, Roiser J, Cools R, Rubinszstein D, Sahakian B, Robbins T. 2005. Stop signal response inhibition is not modulated by tryptophan depletion or the serotonin transporter polymorphism in healthy volunteers: implications for the 5-HT theory of impulsivity. Psychopharmacology 182:570-578.

Conner TST, Jensen KKP, Tennen H, Furneaux HM, Kranzler HR, Covault J. 2010. Functional polymorphisms in the serotonin 1B receptor gene (HTR1B) predict self reported anger and hostility among young men. Am J Med Genet 153B:67-78.

Cools Roshan, Roberts AC, Robbins TW. 2008. Serotoninergic regulation of emotional and behavioural control processes. Trends Cogn Sci 12:31-40.

Crosbie J, Arnold P, Paterson A, Swanson J, Dupuis A, Li X, et al. 2013. Response inhibition and ADHD traits: correlates and heritability in a community sample. J Abnorm Child Psychol 41:497-507.

Duan J, Sanders AR, Molen JE Vander, Martinolich L, Mowry B J, et al. 2003. Polymorphisms in the 5'-untranslated region of the human serotonin receptor 1B (HTR1B) gene affect gene expression. Mol Psychiatry 8:901-910.

Durston S, Mulder M, Casey BJ, Ziermans T, Van Engeland H. 2006. Activation in ventral prefrontal cortex is sensitive to genetic vulnerability for attention-deficit hyperactivity disorder. Biol Psychiatry 60:1062-1070.

Eagle DM, Lehmann O, Theobald DEH, Pena Y, Zakaria R, Ghosh R, et al. 2009. Serotonin depletion impairs waiting but not stop-signal reaction time in rats: implications for theories of the role of 5-HT in behavioral inhibition. Neuropsychopharmacology 34:1311-1321.

Esterman M, Rosenberg MD, Noonan SK. 2014. Intrinsic fluctuations in sustained attention and distractor processing. J Neurosci 34:1724-1730.

Fassbender C, Murphy K, Hester R, Meaney J, Robertson IH, Garavan H. 2006. The role of a right fronto-parietal network in cognitive control: Common activations for "cues-to-attend" and response inhibition. J Psychophysiol 20:286-296.

Finger EC, Marsh AA, Buzas B, Kamel N, Rhodes R, Vythilingham M, et al. 2007. The impact of tryptophan depletion and 5-HTTLPR genotype on passive avoidance and response reversal instrumental learning tasks. Neuropsychopharmacology 32:206-215.

Gizer IR, Ficks C, Waldman ID. 2009. Candidate gene studies of ADHD : a meta-analytic review. Hum Genet 126:51-90. 
Goos LM, Crosbie Jennifer, Payne S, Schachar R. 2009. Validation and extension of the endophenotype model in ADHD patterns of inheritance in a family study of inhibitory control. Am J Psychiatry 166:711-717.

Hayes AF. 2013. Introduction to mediation, moderation, and conditional process analysis: a regression-based approach. London: Guilford Press.

Heinz A, Jones DW, Mazzanti C, Goldman D, Ragan P, Hommer D, et al. 2000. A relationship between serotonin transporter genotype and in vivo protein expression and alcohol neurotoxicity. Biol Psychiatry 47:643-649.

Holm S. 1979. A simple sequentially rejective multiple test procedure. Scand J Stat 6:65-70.

HuangY, Oquendo MA, Friedman JMH, Greenhill LL, Brodsky B, Malone KM, et al. 2003. Substance abuse disorder and major depression are associated with the human 5-HT1B receptor gene (HTR1B) G861C polymorphism. Neuropsychopharmacology 28:163-169.

Kendler KS, Neale MC. 2010. Endophenotype: a conceptual analysis. Mol Psychiatry 15:789-97.

Landaas ET, Johansson S, Jacobsen KK, Ribasés M, Bosch R, Sánchez-Mora C, et al. 2010. An international multicenter association study of the serotonin transporter gene in persistent ADHD. Genes Brain Behav 9:4494-4458.

Lee J-H, Kim H-T, Hyun D-S. 2003. Possible association between serotonin transporter promoter region polymorphism and impulsivity in Koreans. Psychiatry Res 118:19-24.

Lee SH, Ripke S, Neale BM, Faraone Stephen V, Purcell SM, Perlis RH, et al. 2013. Genetic relationship between five psychiatric disorders estimated from genome-wide SNPs. Nat Genet 45:984-994.

Lesch KP, Bengel D, Heils A, Sabol SZ, Greenberg BD, Petri S, et al. 1996. Association of anxiety-related traits with a polymorphism in the serotonin transporter gene regulatory region. Science 274:1527-1531.

Logan GD, Cowan WB, Davis KA. 1984. On the ability to inhibit simple and choice reaction time responses: a model and a method. J Exp Psychol Hum Percept Perform 10:276-291.

Van Meel CS, Heslenfeld DJ, Oosterlaan J, Sergeant JA. 2007. Adaptive control deficits in attention-deficit/hyperactivity disorder (ADHD): the role of error processing. Psychiatry Res 151:211-220.

Murphy TM, Ryan M, FosterT, Kelly C, McClelland R, O'Grady J, et al. 2011. Risk and protective genetic variants in suicidal behaviour: association with SLC1A2, SLC1A3, 5-HTR1B \& NTRK2 polymorphisms. Behav Brain Funct 7:22.

Nigg JT. 2000. On inhibition/disinhibition in developmental psychopathology: views from cognitive and personality psychology and a working inhibition taxonomy. Psychol Bull 126:220-246.

Oades RD. 2008. Dopamine-serotonin interactions in attentiondeficit hyperactivity disorder (ADHD). Prog. Brain Res 172:543-565.

\section{Supplementary materials available online}

\section{Supplementary methods}

Determining diagnostic status in the NeuroIMAGE sample

Participant inclusion

Genotyping

fMRI acquisition and preprocessing

fMRI single subject analysis
Rubia K, Lee F, Cleare AJ, Tunstall N, Fu CHY, Brammer M, et al. 2005. Tryptophan depletion reduces right inferior prefrontal activation during response inhibition in fast, eventrelated fMRI. Psychopharmacology 179:791-803.

Rubia K, Smith AB, Taylor E, Brammer M. 2007. Linear agecorrelated functional development of right inferior fronto-striato-cerebellar networks during response inhibition and anterior cingulate during error-related processes. Hum Brain Mapp 28:1163-1177.

Sanders AR, Cao Q, Taylor J, Levin TE, Badner JA, Cravchik A, et al. 2001. Genetic diversity of the human serotonin receptor 1B (HTR1B) gene. Genomics 72:1-14.

Stoltenberg SF, Glass JM, Chermack ST, Flynn HA, Li S, Weston ME, Burmeister M. 2006. Possible association between response inhibition and a variant in the brain-expressed tryptophan hydroxylase-2 gene. Psychiatr Genet 16:35-38.

Strobel A, Dreisbach G, Müller J, Goschke T, Brocke B, Lesch Klaus-Peter. 2007. Genetic variation of serotonin function and cognitive control. J Cogn Neurosci 19:1923-1931.

Varga G, Szekely A, Antal P, Sarkozy P, Nemoda Z, Demetrovics Z, et al. 2012. Additive effects of serotonergic and dopaminergic polymorphisms on trait impulsivity. Am J Med Genet 159B: 281-288.

Von Rhein D, Mennes M, Van Ewijk H, Groenman AP, Zwiers M, Oosterlaan J, et al. 2014. The NeuroIMAGE study: a prospective phenotypic, cognitive, genetic and MRI study in children with attention-deficit hyperactivity disorder. Design and descriptives. Eur Child Adolesc Psychiatry Epub ahead.

Van Rooij D, Hartman CA, Mennes M, Oosterlaan J, Franke Barbara, Rommelse N, et al. 2015a. Distinguishing adolescents with ADHD from their unaffected siblings and healthy comparison subjects by neural activation patterns during response inhibition. Am J Psychiatry epub ahead.

Van Rooij D, Hartman CA, Mennes M, Oosterlaan J, Franke Barbara, Rommelse N, et al. 2015b. Altered neural connectivity patterns during response inhibition in adolescents with ADHD and their unaffected siblings. NeuroImage: Clinical 7:325-335.

Walderhaug E, Herman AI, Magnusson A, Morgan MJ, Landrø NI. 2010. The short (S) allele of the serotonin transporter polymorphism and acute tryptophan depletion both increase impulsivity in men. Neurosci Lett 473:208-211.

Walderhaug E, Lunde H, Nordvik JE, Landrø NI, Refsum H, Magnusson A. 2002. Lowering of serotonin by rapid tryptophan depletion increases impulsiveness in normal individuals. Psychopharmacology 164:385-391.

Winstanley CA, Eagle DM, Robbins TW. 2006. Behavioral models of impulsivity in relation to ADHD: translation between clinical and preclinical studies. Clin Psychol Rev 26:379-395.

Woo C-W, Krishnan A, Wager TD. 2014. Cluster-extent based thresholding in fMRI analyses: pitfalls and recommendations. Neuroimage 91:412-419.

\section{Supplementary results \\ fMRI task activation \\ Between group differences in $F M R I$ activation}

Genetic effects on between-group differences in fMRI activation

Role of genetic effects in whole-brain fMRI activation and stop-task performance

Supplementary Tables 1-5.

Supplementary Figures 1-3. Available online at http://dx.doi.org/10.3109/15622975.2015.1067371. 